\title{
Severe pet-transmitted zoonosis in a patient with a compromised immune system
}

\author{
Marc Bienz MD MSc, Marcel Tomaszewski MD, Emily G. McDonald MD MSc
}

Cite as: CMAJ 2018 November 12;190:E1332-6. doi: 10.1503/cmaj.180720

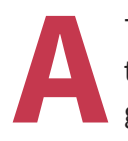

76-year-old man originally from Barbados was admitted to hospital after experiencing one month of progressive generalized weakness that culminated in his inability to carry out independent activities of daily living. The patient's medical history included hypertension, type 2 diabetes, previous smoking (20 pack-years) and diabetic nephropathy that resulted in a kidney transplant six years earlier. After transplantation surgery, the patient had experienced stage 5 chronic renal insufficiency as a result of progressive allograft dysfunction. The patient was taking an immune suppression regimen consisting of extended release tacrolimus ( $25 \mathrm{mg} / \mathrm{d})$, mycophenolate mofetil (720 mg, twice daily) and prednisone $(5 \mathrm{mg} / \mathrm{d}$ ). Other medications taken by the patient included labetolol, amlodipine, calcitriol and darbapoietin $\alpha$.

The patient reported having a decreased appetite and undergoing a 6-kg weight loss during the previous month, without fever, chills or night sweats. Several small painless peripheral nodular skin lesions had also developed over a two-month period. The patient reported no other symptoms during the review of systems. He had not recently travelled outside of Quebec and had no sick contacts. However, he owned several pet fish, which he kept in a fresh water aquarium that he cleaned himself twice each month.

On initial physical examination, the patient had no fever and had normal vital signs. He was alert and oriented, with no lateralizing signs. He had temporal muscle wasting and a body mass index of $19 \mathrm{~kg} / \mathrm{m}^{2}$. We found six ulcerated lesions, the largest of which was $2 \mathrm{~cm}$ in diameter, on the dorsal hand, forearm and legs (Figure 1). We felt no lymphadenopathy or hepatosplenomegaly. The results of initial laboratory investigations are presented in Box 1.

We biopsied a skin lesion on the patient's left forearm, and microbiological testing showed acid-fast bacilli. Radiography of the patient's chest showed bilateral nodular densities, and computed tomography confirmed the presence of multiple pulmonary nodules (Figure 2). Gastroscopy showed multiple millimetric black mucosal discolourations in the duodenum (Figure $3 \mathrm{~A}$ and 3B). The lesions were thought to be evidence of disseminated mycobacterium to the gastrointestinal tract. The patient was

\section{KEY POINTS}

- Zoonotic infections can be life-threatening for patients with compromised immune systems; knowing the mechanisms of transmission and avoiding exposure to pets with a high risk of transmission can help prevent their acquisition.

- Patients with a high risk of acquiring a zoonotic infection include patients with HIV, patients with a solid or hematologic tumour, and patients who are receiving certain immunesuppressive medications, either alone or in combination.

- In the absence of prevention, early recognition of a zoonotic infection and directed antimicrobial therapy may substantially improve outcomes.

- Physicians and other health care practitioners have an important role in educating patients and family members regarding measures to prevent pet-transmitted infections.

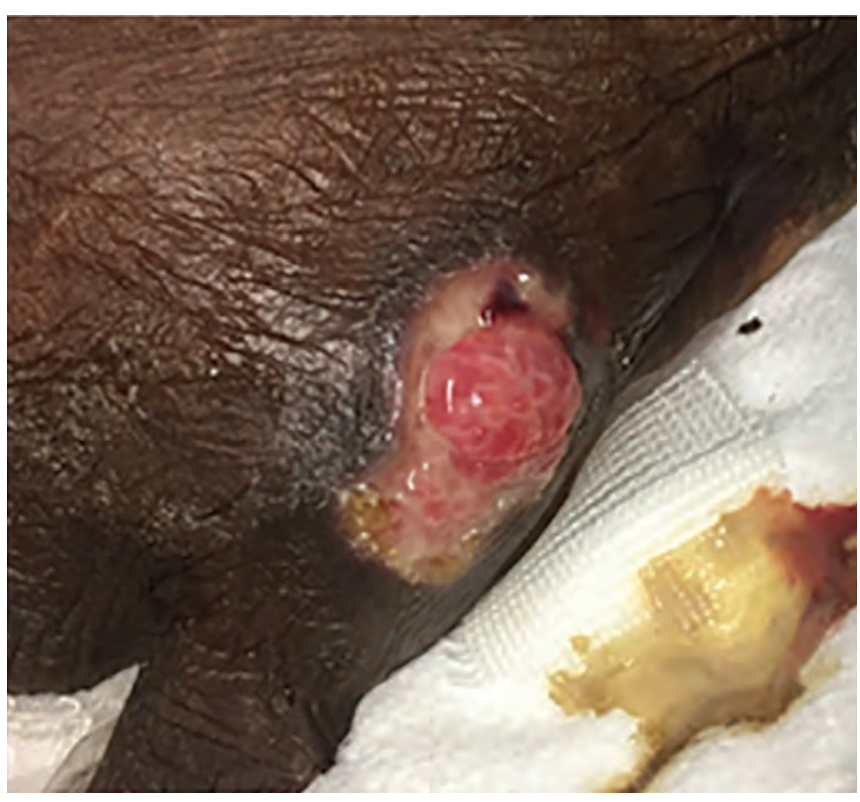

Figure 1: Granulomatous lesion on left hand of a 76-year-old man who presented with progressive generalized weakness. Five similar lesions were present on his forearms and legs. 
Box 1: Results of laboratory investigations

\begin{tabular}{|c|c|c|c|}
\hline Investigation & Value during hospital stay & Baseline value & Normal range \\
\hline Hemoglobin, g/L & 82 & 90 & $135-175$ \\
\hline Platelets, $\times 10^{9} / \mathrm{L}$ & 87 & 180 & $140-450$ \\
\hline White blood cells, $\times 10^{9} / \mathrm{L}$ & 3.9 & 5.0 & $4.50-11.00$ \\
\hline Absolute neutrophil count, $\times 10^{9} / \mathrm{L}$ & 3.67 & 3.70 & $1.80-7.70$ \\
\hline Absolute lymphocyte count & 0.03 & 0.04 & $1.00-4.80$ \\
\hline Absolute eosinophil count, $\times 10^{9} / \mathrm{L}$ & 0.02 & 1.3 & $0.00-0.80$ \\
\hline Creatinine, $\mu \mathrm{mol} / \mathrm{L}$ & 375 & 200 & $55-110$ \\
\hline Ferritin, $\mu \mathrm{g} / \mathrm{L}$ & 911 & 130 & $23.9-366.0$ \\
\hline Albumin, $g / L$ & 21 & 30 & $38-52$ \\
\hline C-reactive protein, $\mathrm{mg} / \mathrm{L}$ & 213.5 & - & $0.0-5.0$ \\
\hline HIV antigen & Negative & Negative & - \\
\hline CMV PCR, copies/mL & Not detected & & - \\
\hline \multicolumn{4}{|l|}{ Microbiology cultures } \\
\hline Bacterial blood & Negative $\times 4$ & & \\
\hline Mycobacterium blood & Mycobacterium marinum & & \\
\hline Bacterial sputum (first) & Yeast & & \\
\hline Bacterial sputum (second) & Pseudomonas aeroginosa & & \\
\hline Wound bacterial (first) & $\begin{array}{l}\text { Staphylococcus aureus } \\
\text { Enterobacter cloacae complex }\end{array}$ & & \\
\hline Wound bacterial (second) & Staphylococcus aureus & & \\
\hline Wound bacterial (third and fourth) & Pseudomonas aeroginosa & & \\
\hline Deep fungal bronchoalveolar lavage & Candida albicans & & \\
\hline Deep mycobacterium bronchoalveolar lavage & Mycobacterium marinum & & \\
\hline Cerebrospinal fluid mycobacterium & Negative $\times 2$ & & \\
\hline Stool bacterial & Pseudomonas aeroginosa & & \\
\hline Stool ova and parasite & Negative & & \\
\hline
\end{tabular}

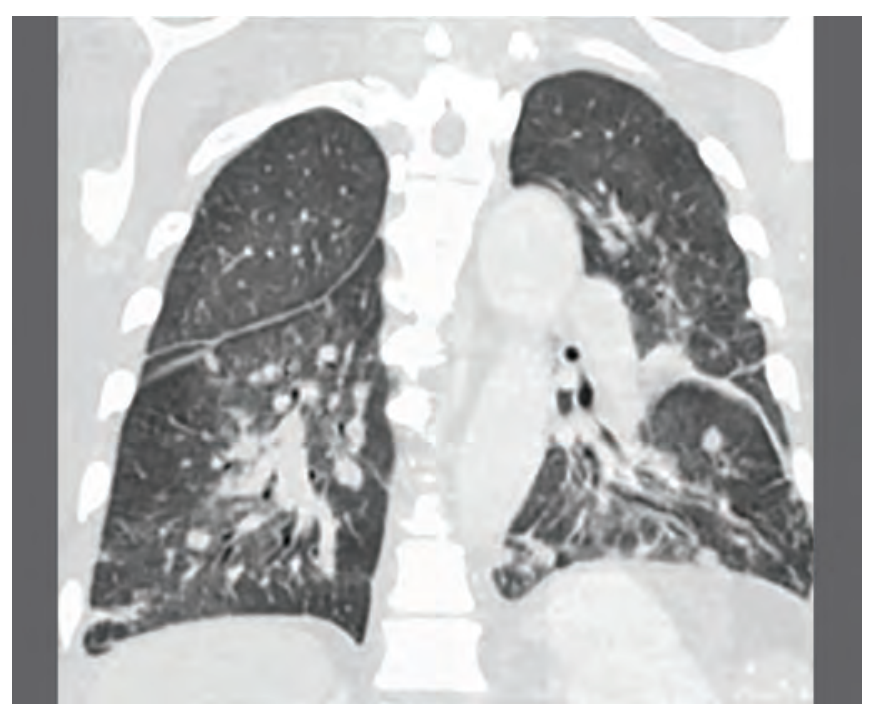

Figure 2: Computed tomography of the chest showing large nodular opacities and diffuse ground glass opacities. started on empirical treatment consisting of imipenem/cilastin, tigecycline and rifampin. Tuberculosis cultures of the blood showed the presence of Mycobacterium marinum within seven days. Extended treatment with monocycline and rifampin was started. In addition, bronchoalveolar lavage fluid grew Mycobacterium marinum within three weeks. Fish tank maintenance was considered the culprit source, resulting in disseminated Mycobacterium marinum infection in our patient who had a compromised immune system.

Despite receiving appropriate antimycobacterial therapy to which his skin lesions responded, the patient remained deconditioned from a prolonged stay in hospital. He had progressive allograft failure and required intermittent hemodialysis. His stay was further complicated by an asystolic cardiac arrest, with subsequent anoxic brain injury, and multisystem organ failure that resulted in his death. An autopsy confirmed disseminated Mycobacterium marinum infection of mediastinal, hilar and subcarinal lymph nodes, the proximal small intestinal mucosa, spleen, liver, lungs and skin (Figure 4). 


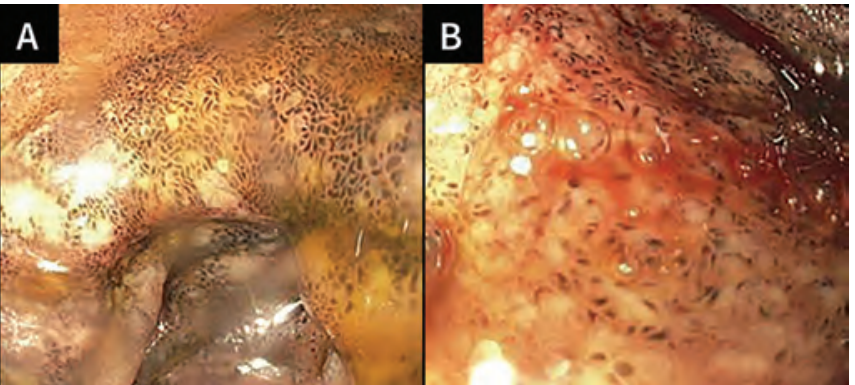

Figure 3: (A) Diffuse millimetric black discoloration of the duodenal mucosa found on gastroscopy; (B) close-up view.

\section{Discussion}

The accurate diagnosis of zoonotic infections can be challenging in patients with compromised immune systems owing to the uncommon nature of many of these infections and presenting symptoms that may be subtle during early stages of infection. Our patient's case exemplifies how zoonotic infections can rapidly become life-threatening in this population. Household pets, such as reptiles, fish and birds, are increasingly encountered in North American households. ${ }^{1}$ As a greater number of patients are exposed to immune suppression, the possibility of patients with compromised immune systems interacting with pets increases. Among more than 200 known zoonotic infections, 40 are transmitted by pets. ${ }^{2}$ Thus, patients should be properly informed regarding general measures to prevent transmission.

\section{Risks of zoonosis transmission}

Medical conditions and therapies can result in immune deficiency. High-risk immune-compromising conditions include solid organ and hematologic cancers, transplants, HIV infection, congenital immunodeficiencies, and asplenia or splenectomy. ${ }^{3}$ High-risk medical treatments include high-dose steroids (> $20 \mathrm{mg} / \mathrm{d}$ prednisone for $\geq 4 \mathrm{wk}$ ), chemotherapy and radiation therapy, tumour necrosis factor inhibitors, antimetabolites, T cell-depleting agents, interleukin-2 receptor antagonists, calcineurin inhibitors and mammalian target of rapamycin inhibitors (Box 2).

Various immunosuppressive drugs and disease states have differential effects on $B$ and $T$ cell function, in addition to complement inhibition. For this reason, not all states of immune deficiency are equal in terms of risk of infection. Data on specific infectious complications associated with certain drug classes prescribed after solid organ transplantation are available through antimicrobe.org. ${ }^{4}$

Regarding nontuberculous mycobacteria, higher rates of infection are associated with loss of $\mathrm{T}$ cell function, decreased production of interferon or tumour necrosis factor $\alpha$ (TNF $\alpha$ ), and specific therapies such as moderate to high doses of steroids (> $10 \mathrm{mg} / \mathrm{d}$ prednisone), medications targeting $T N F \alpha$, interleukin-12/23, or Janus kinase (JAK), 5,6 and mycophenolate mofetil-containing immune suppression regimens. ${ }^{6}$

Exposure to pets is common for patients with immunecompromising conditions. In one study, pets were found in $55 \%$ of households that included a member with an immunecompromising condition. ${ }^{1}$ High-risk pets, identified based on higher transmission rates, should be avoided. These pets include cats and dogs aged 6 months or younger, reptiles and amphibians, rodents and young poultry. ${ }^{7}$ In a study that involved children with cancer, $77 \%$ of pets acquired shortly after the diagnosis of cancer were high risk. ${ }^{7}$

Certain work environments and public spaces increase the risk of transmission of zoonotic infections (Box 2). Pet shop employees may contract salmonellosis or psittacosis from birds and rodents. ${ }^{8}$ Veterinary clinics are associated with the transmission of Bartonella, Brucella, methicillin-resistant Staphylococcus aureus and Salmonella. ${ }^{9,10}$ Exposure to cattle on farms can lead to cryptosporidiosis, ${ }^{10}$ and petting zoo animals should be avoided given their potential for colonization with Salmonella and Campylobacter. ${ }^{2}$ Additional infectious vectors that are important to consider include plants, fresh flowers and certain foods. ${ }^{10}$

Physicians play an important role in counselling patients on the risk of zoonosis. Stull and colleagues showed that physicians query pet-ownership in less than $50 \%$ of pet-owning families of children with cancer. ${ }^{7}$ Routine counselling tailored to a patient's exposure may help with early recognition and prompt treatment of these infections. Veterinary staff are also potential information providers for pet owners with compromised immune systems. ${ }^{3}$

\section{Measures to prevent transmission}

In 2010, the Centers for Disease Control and Prevention (CDC) published guidelines for the prevention of opportunistic
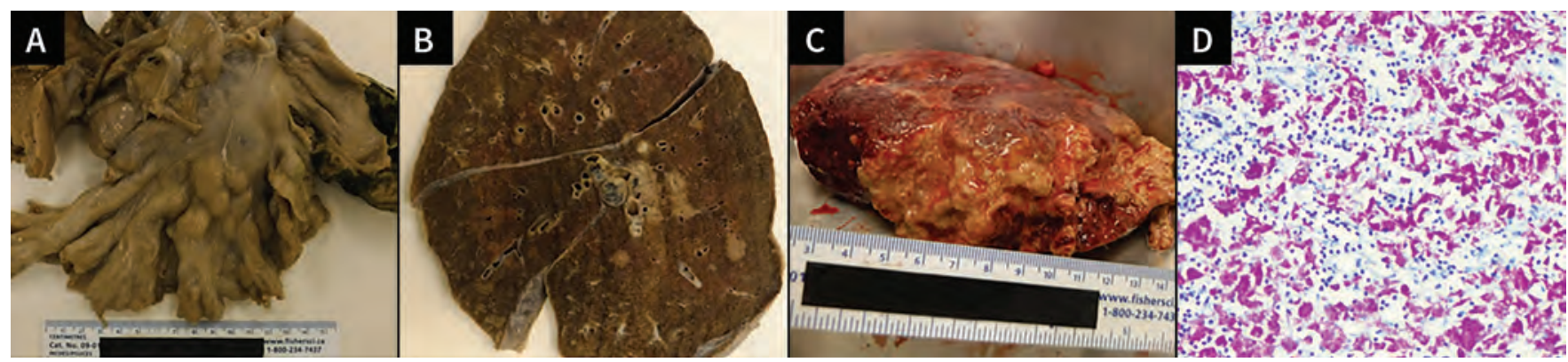

Figure 4: Images taken postmortem showing (A) diffuse large mesenteric nectroctic lymphadenopathy in the duodenum and jejunum, (B) bilateral mediastinal, hilar and subcarinal lymphadenopathy and pale brown non-caseating nodules in the lung, (C) massive necrosis of the spleen and (D) Mycobacterium infection (dark blue) of a lymph node (original magnification $\times 400$ ). 
Box 2: Predisposing risk factors for zoonotic infections ${ }^{2,3,5-10}$

- Immune-compromising conditions

- Solid organ and hematologic cancers

- Receiving a transplant

- HIV infection

- Congenital immunodeficiencies

- Splenectomy or asplenia

- Immune-suppressive therapies

- High-dose steroids (> $20 \mathrm{mg} / \mathrm{d}$ prednisone, for $\geq 4 \mathrm{wk}$ )

- Chemotherapy and radiation therapy

- Tumour necrosis factor inhibitors (i.e., infliximab, adalimumab, etanercept)

- Antimetabolites (i.e., methotrexate, azathioprine, mercaptopurine)

- T cell-depleting agents (i.e., thymoglobulin, muromonab-CD3, alemtuzumab)

- Interleukin-2 receptor antagonists (i.e., daclizumab, basiliximab)

- Calcineurin inhibitors (i.e., tacrolimus, cyclosporine)

- Mammalian target of rapamycin inhibitors (i.e., sirolimus)

- High-risk pets

- Cats and dogs aged 6 months or younger

- Reptiles and amphibians

- Rodents

- Young poultry

- High-risk environments

- Pet shops

- Veterinary clinics

- Farms

- Petting zoos

infections in transplant recipients. ${ }^{10}$ This is a helpful reference for all at-risk patients and contains recommendations on pet safety and the prevention of zoonotic infections. In addition, a comprehensive list of most zoonotic infections, their mode of transmission and their animal reservoir has been published by Kotton and colleagues. ${ }^{11}$

Educating patients on the mechanisms of transmission can help promote safer habits and subsequently decrease infection rates. The following suggestions are compatible with the CDC guidelines and other publications aimed at preventing infection in this population (Box 3). Contact transmission from pets' skin, saliva, urine and feces through bites and scratches is common. ${ }^{2}$ In addition, aerosol and droplets can transmit Bordetella species from dogs and Cryptococcus neoformans from birds. ${ }^{11}$ Therefore, general preventative measures such as thorough handwashing after handling of pets, avoiding contact with feces and having someone else clean habitats such as bird cages, fish tanks and litter boxes should be encouraged. If exposure cannot be avoided, gloves and possibly a surgical mask are recommended. ${ }^{12}$ Litter boxes should be changed daily and kept in more secluded locations to reduce the risk of Toxoplasmosis gondii contamination. ${ }^{10}$
Box 3: Safe pet-handling recommendations ${ }^{2,10,11-13}$

- Ensure thorough handwashing after contact with pets and their body fluids; avoid contact with pet feces.

- Cleaning of habitats (cages, fish tanks, litter boxes) should not be performed by the patient; gloves and a surgical mask can be worn if exposure cannot be avoided.

- Litter boxes should be changed daily and kept in secluded locations.

- Avoid scratches and bites, and protect wounds and other portals of entry from contact with pets.

- Pets should not be fed raw meat or eggs and should drink only clean water.

- Stray animals should not be brought into the home, and cats should stay indoors.

- All pets should be vaccinated and have regular visits with the veterinarian.

Handling cats and dogs should be done with care to avoid scratches and bites, and cat nails should be kept short. ${ }^{12}$ Saliva can transmit Staphylococcus species from dogs, and Bartonella henselae or Pasturella multilocida from cats. Capnocytophaga canimorsus infection from dog bites is uncommon, but can rapidly progress to shock and death. ${ }^{2}$ Handling of rodents, fish and reptiles can transmit Salmonella and Campylobacter. ${ }^{11}$

Patients should be reminded that wounds, venous catheters, rashes and mucositis are examples of high-risk skin barrier rupture. All wounds and portals of entry should be protected from contact with animals and their habitat. Mycobacterium marinum can be acquired from fish tanks through skin-barrier rupture, even in patients with competent immune systems, and may start as a single small lesion, before progressing to more severe, disseminated disease. ${ }^{11,13}$

Finally, pets should not be fed raw meat or eggs, and should only be allowed to drink clean water (not toilet water). Stray animals should not be brought into the home, and cats should stay indoors. As a general rule, sick pets should be brought to the veterinarian, and all pets should undergo routine vaccination. ${ }^{10}$

\section{Conclusion}

Early recognition and prompt initiation of directed antimicrobial therapy can substantially affect the outcome of pet-transmitted zoonotic infections in patients with compromised immune systems. As shown in our patient's case, some zoonotic infections can disseminate and have a life-threatening course. Despite a quick diagnosis and targeted therapy, disseminated Mycobacterium marinum infection contributed to the death of our patient. Patient education on preventing pet-transmitted infections should be the standard of care for patients at risk of zoonosis because of a compromised immune system.

\section{References}

1. Stull JW, Peregrine AS, Sargeant JM, et al. Household knowledge, attitudes and practices related to pet contact and associated zoonoses in Ontario, Canada. BMC Public Health 2012;12:553.

2. Steele RW. Should immunocompromised patients have pets? Ochsner J 2008;8:134-9. 
3. Stull JW, Stevenson KB. Zoonotic disease risks for immunocompromised and other high-risk clients and staff: promoting safe pet ownership and contact. Vet Clin North Am Small Anim Pract 2015;45:377-92, vii.

4. Burdette SD, Sun H-Y. Infections associated with specific immunosuppressive agents in transplant recipients. Pittsburgh: Antimicrobe; 2010-2017. Available: www.antimicrobe.org/new/t34_dw.html (accessed 2018 June 20).

5. Lake MA, Ambrose LR, Lipman MC, et al. "Why me, why now?" Using clinical immunology and epidemiology to explain who gets nontuberculous mycobacterial infection. BMC Med 2016;14:54.

6. Brode SK, Jamieson FB, Ng R, et al. Increased risk of mycobacterial infections associated with anti-rheumatic medications. Thorax 2015;70:677-82.

7. Stull JW, Brophy J, Sargeant JM, et al. Knowledge, attitudes, and practices related to pet contact by immunocompromised children with cancer and immunocompetent children with diabetes. J Pediatr 2014;165:348-55.e2.

8. Halsby KD, Walsh AL, Campbell C, et al. Healthy animals, healthy people: zoonosis risk from animal contact in pet shops, a systematic review of the literature. PLOS One 2014;9:e89309.

9. Weese JS, Dick H, Willey BM, et al. Suspected transmission of methicillin-resistant Staphylococcus aureus between domestic pets and humans in veterinary clinics and in the household. Vet Microbiol 2006;115:148-55.

10. Centers for Disease Control and Prevention; Infectious Disease Society of America; American Society of Blood and Marrow Transplantation. Guidelines for preventing opportunistic infections among hematopoietic stem cell transplant recipients. MMWR Recomm Rep 2000;49:1-125, CE1-7.

11. Kotton CN. Zoonoses in solid-organ and hematopoietic stem cell transplant recipients. Clin Infect Dis 2007;44:857-66.

12. Stull JW, Brophy J, Weese JS. Reducing the risk of pet-associated zoonotic infections. CMAJ 2015;187:736-43.

13. Fleming P, Keystone JS. Mycobacterium marinum infection with sporotrichoid spread from fish tank exposure. CMAJ 2017;189:E76.

\section{Competing interests: None declared.}

This article has been peer reviewed.

The authors have obtained patient consent.

Affiliations: Department of Medicine (Bienz, Tomaszewski, McDonald), McGill University Health Centre; Clinical Practice Assessment Unit (McDonald), Montréal, Que.

Contributors: All authors participated in the conception, design, writing and revision of the article. All authors approved the final version of the manuscript to be published and agreed to act as guarantors of the results.

Acknowledgements: The authors thank Dr. Todd C. Lee for his opinions and expert review of this manuscript, and Dr. Issa-Chergui and Dr. Khadang for providing images from the autopsy.

Correspondence to: Marc Bienz, marc.bienz@mail.mcgill.ca

The section Cases presents brief case reports that convey clear, practical lessons. Preference is given to common presentations of important rare conditions, and important unusual presentations of common problems. Articles start with a case presentation (500 words maximum), and a discussion of the underlying condition follows (1000 words maximum). Visual elements (e.g., tables of the differential diagnosis, clinical features or diagnostic approach) are encouraged. Consent from patients for publication of their story is a necessity. See information for authors at www.cmaj.ca. 